\title{
Solved and Unsolved Problems
}

\section{Michael Th. Rassias (University of Zürich, Switzerland)}

Number theorists are like lotus-eaters - having tasted this food they can never give it up.

Leopold Kronecker (1823-1891)

The present column is devoted to Analytic Number Theory. For the previous "Solved and Unsolved Problems" column devoted to Number Theory, the interested reader is referred to the Issue 103, March 2017, of the EMS Newsletter.

\section{Six new problems - solutions solicited}

218.

Determine the sum of the series

$$
\sum_{n=1}^{\infty} \frac{\varphi(n)}{2^{n}-1},
$$

where $\varphi$ is the Euler's totient function.

(Dorin Andrica, Babeş-Bolyai University,

Cluj-Napoca, Romania)

219 . Let $\omega(n)$ denote the number of distinct prime factors of a non-zero natural number $n$.

(i) Prove that $\sum_{n \leq x} \omega(n)=x \log \log x+O(x)$.

(ii) Prove that $\sum_{n \leq x} \omega(n)^{2}=x(\log \log x)^{2}+O(x \log \log x)$.

(iii) Using (i) and (ii), prove that $\sum_{n \leq x}(\omega(n)-\log \log x)^{2}=$ $O(x \log \log x)$.

(iv) Using (iii), prove that $\sum_{n \leq x}(\omega(n)-\log \log n)^{2}=$ $O(x \log \log x)$.

(v) Using (iv), prove that $\omega(n)$ has normal order $\log \log n$, i.e., for every $\varepsilon>0$,

$$
\begin{aligned}
& \#\{n \leq x:(1-\varepsilon) \log \log n<\omega(n) \\
&<(1+\varepsilon) \log \log n\} \sim x \quad(\text { as } x \rightarrow \infty) .
\end{aligned}
$$

a. Parts (i)-(v) of Problem 219 are extracted from a proof by Paul Turán (1910-1976), published in 1934, of a theorem of G. H. Hardy (18771947) and S. Ramanujan (1887-1920), published in 1917; see references below:

[1] G. H. Hardy and S. Ramanujan, The normal number of prime factors of a number n. Quart. J. Math. 48 (1917), 76-92.

[2] P. Turán, On a Theorem of Hardy and Ramanujan. J. London Math. Soc. 9 (1934), 274-276.

(Alina Carmen Cojocaru, Department of Mathematics, Statistics and Computer Science, University of Illinois at Chicago, USA, and Institute of Mathematics "Simion Stoilow" of the Romanian Academy, Bucharest, Romania)
220. Using Chebyshev's Theorem, prove that for any integer $M$ there exists an even integer $2 k$ such that there are at least $M$ primes $p$ with $p+2 k$ also prime. Unfortunately $2 k$ will depend on $M$. If it did not, we would have solved the Twin Prime Conjecture, namely, there are infinitely many primes $p$ such that $p+2$ is also prime.

(Steven J. Miller, Department of Mathematics \& Statistics, Williams College, Massachusetts, USA)

221. For any three integers $a, b, c$, with $\operatorname{gcd}(a, b, c)=1$, prove that there exists an integer $m$ such that

$$
0 \leq m \leq 2^{2^{2002}} c^{\frac{1}{1000}} \text { and } \operatorname{gcd}(a+m b, c)=1 .
$$

(Abhishek Saha, School of Mathematical Sciences, Queen Mary University of London, UK)

\section{Show that}

$$
\begin{array}{ll}
\sum_{n=1}^{\infty} \frac{\sin ^{2}(\pi \delta n)}{n^{2}}=\frac{1}{2} \pi^{2} \delta(1-\delta) & \text { for } 0 \leq \delta \leq 1, \\
\sum_{n=1}^{\infty} \frac{\sin ^{3}(\pi \delta n)}{n^{3}}=\frac{1}{2} \pi^{3} \delta^{2}\left(\frac{3}{4}-\delta\right) & \text { for } 0 \leq \delta \leq 1 / 2, \\
\sum_{n=1}^{\infty} \frac{\sin ^{4}(\pi \delta n)}{n^{4}}=\frac{1}{2} \pi^{4} \delta^{3}\left(\frac{2}{3}-\delta\right) & \text { for } 0 \leq \delta \leq 1 / 2 .
\end{array}
$$

Setting $\delta=1 / 2$, deduce the values of $\zeta(2)$ and $\zeta(4)$.

(Olof Sisask, Department of Mathematics, Stockholm University, Sweden)

223. Fix a prime number $p$, and an integer $\beta \geq 2$. Consider the function defined on $x \in \mathbf{R}$ by $e(x)=\exp (2 \pi i x)$. Given a coprime residue class $r \bmod p^{\beta}$, consider the additive character defined on integers $m \in \mathbf{Z}$ by $m \mapsto e\left(\frac{m r}{p^{\beta}}\right)$. Given a complex parameter $s \in \mathbf{C}$ with $\operatorname{Re}(s)>1$, consider the Dirichlet series defined by

$$
D\left(s, r, p^{\beta}\right)=\sum_{m \geq 1} e\left(\frac{r m}{p^{\beta}}\right) m^{-s} .
$$

Show that this series has an analytic continuation to all $s \in \mathbf{C}$, and moreover that it satisfies a functional equation relating values at $s$ to $1-s$.

(Jeanine Van Order, Fakultät für Mathematik, Universität Bielefeld, Germany.)

II (A) An Open Problem, by Joseph Najnudel (School of Mathematics, University of Bristol, UK)

\section{Central limit theorems for random multiplicative functions}

The distribution of the patterns obtained by taking consecutive values of arithmetic multiplicative functions have been intensively stud- 
ied. For example, a conjecture by Chowla [2] states that for all $k \geq 1$, each possible sign pattern of

$$
(\lambda(n+1), \ldots, \lambda(n+k))
$$

appears with asymptotic density $2^{-k}, \lambda$ being the Liouville function, i.e.,

$$
\lambda(m)=(-1)^{\Omega(m)}
$$

where $\Omega(m)$ is the number of prime factors of $m$, counted with multiplicity. It has been proven by Hildebrand [7] that for $k=3$, the eight possible values of

$$
(\lambda(n+1), \lambda(n+2), \lambda(n+3))
$$

appear infinitely often. This result has been improved by Matomäki, Radziwill and Tao [8], who prove that these eight values appear with a positive lower density. Similar results and conjectures are stated for the Möbius function, or for the number of prime factors modulo 3 (see [10]).

In [9], we consider similar questions for consecutive values of random completely multiplicative functions $\left(X_{m}\right)_{m \geq 1}$, such that $\left(X_{p}\right)_{p}$ prime are i.i.d. random variables on the unit circle $\mathbb{U}$, and

$$
X_{m}=\prod_{p \text { prime }} X_{p}^{v_{p}(m)}
$$

where $v_{p}(m)$ is the $p$-adic valuation of $m$. In this setting, the law of $\left(X_{m}\right)_{m \geq 1}$ is completely determined by the law of $X_{2}$, and we have studied the two following cases in detail: $X_{2}$ uniform on the unit circle $\mathbb{U}$, and $X_{2}$ uniform on the set $\mathbb{U}_{q}$ of $q$-th roots of unity for some integer $q \geq 2$. The randomness we have introduced allows us to prove more precise results than what is known for Liouville or Möbius function, with much more elementary proofs. However, a part of the arguments used in [9] are related to deep results on the number and the size of the solutions of some diophantine equations. The main result of [9] is the proof that the empirical distribution

$$
\frac{1}{N} \sum_{n=1}^{N} \delta_{\left(X_{n+1}, \ldots, X_{n+k}\right)}
$$

tends almost surely to the uniform distribution on $\mathbb{U}^{k}$ if $X_{2}$ is uniform on $\mathbb{U}$, and to the uniform distribution on $\mathbb{U}_{q}^{k}$ if $X_{2}$ is uniform on $\mathbb{U}_{q}$. We also have an estimate on the speed of convergence of the empirical measure: in the case of the uniform distribution on $\mathbb{U}_{q}$, each of the $q^{k}$ possible patterns for $\left(X_{n+1}, \ldots, X_{n+k}\right)$ almost surely occurs with a proportion $q^{-k}+O\left(N^{-t}\right)$ for $n$ running between 1 and $N$, for all $t<1 / 2$. We have a similar result in the uniform case, if the test functions we consider are sufficiently smooth. These results are deduced, via the Fourier transform of the empirical measure, from the following bound, available for all $\left(m_{1}, \ldots, m_{k}\right) \neq(0, \ldots, 0)$ if $X_{2}$ is uniform on $\mathbb{U}$, and for $m_{1}, \ldots, m_{k}$ not all divisible by $q$ if $X_{2}$ is uniform on $\mathbb{U}_{q}$ :

$$
\mathbb{E}\left[\left|\sum_{n=N^{\prime}+1}^{N} \prod_{j=1}^{k} X_{n+j}^{m_{j}}\right|^{2}\right] \leq O\left(\left(N-N^{\prime}\right) N^{\varepsilon}\right),
$$

for $1 \leq N^{\prime}<N$ and $\varepsilon>0$, the implied constant depending only on $k, \varepsilon$, and on $q$ if $X_{2}$ is uniform on $\mathbb{U}_{q}$. The bound is obtained from an upper bound of the number of solutions of

$$
\frac{\prod_{j=1}^{k}\left(n_{1}+j\right)^{m_{j}}}{\prod_{j=1}^{k}\left(n_{2}+j\right)^{m_{j}}} \in \mathcal{A}
$$

where $\mathcal{A}=\{1\}$ if $X_{2}$ is uniform on $\mathbb{U}, \mathcal{A}=\left(\mathbb{Q}_{+}^{*}\right)^{q}$ if $X_{2}$ is uniform on $\mathbb{U}_{q}$.
The convergence (1) corresponds to a law of large numbers satisfied by the sums of the form

$$
\sum_{n=1}^{N} \prod_{j=1}^{k} X_{n+j}^{m_{j}}
$$

An open question concerns the existence of a central limit theorem for such sums. To simplify the discussion, let us focus on the case where $X_{2}$ is uniform on the unit circle. It is not possible to have a central limit theorem for the sum $\sum_{n=1}^{N} X_{n}$, because its $L^{2}$ norm is equal to $n$, whereas its $L^{1}$ norm is $o(\sqrt{n})$, as recently proven by Harper [3]. This last result is called Helson's conjecture (see [6]), and has been previously discussed in several papers including [4] and [5]. However, central limit theorem may be true for other sums. In [1], Chatterjee and Soundararajan have proven a central limit theorem of sums of the form $\sum_{n=N^{\prime}+1}^{N} X_{n}$ when $1 \leq N^{\prime}<N$ and $N^{\prime}$ sufficiently close to $N$. Moreover, in [9], we have quite easily proven the following result: if for all integers $r \geq 1$, the number of non-trivial solutions

$$
\left(n_{1}, \ldots, n_{2 r}\right) \in\{1, \ldots, N\}^{2 r}
$$

of the diophantine equation

$$
\prod_{j=1}^{r} n_{j}\left(n_{j}+1\right)=\prod_{j=1}^{r} n_{r+j}\left(n_{r+j}+1\right)
$$

is negligible with respect to the number of trivial solutions, i.e., $o\left(N^{r}\right)$ when $N \rightarrow \infty$, then we have the central limit theorem:

$$
\frac{1}{\sqrt{N}} \sum_{n=1}^{N} X_{n} X_{n+1} \underset{N \rightarrow \infty}{\longrightarrow} \frac{\mathcal{N}_{1}+i \mathcal{N}_{2}}{\sqrt{2}}
$$

where $\mathcal{N}_{1}$ and $\mathcal{N}_{2}$ are two i.i.d. standard Gaussian variables. This fact is obvious for $r=1$ and we have proven that it is true for $r=2$. More precisely, we have checked that the number of non-trivial solutions of

$$
a(a+1) d(d+1)=b(b+1) c(c+1)
$$

where $1 \leq a<b \leq c<d \leq N$ is between $\delta N-1$ and $N^{3 / 2+o(1)}$, for an explicit constant $\delta>0$. We have also proven that the infimum of the ratio $d / a$ for all the solutions is $3+2 \sqrt{2}$ (this very last result, with elementary but quite difficult solution, may have been suitable for an olympiad problem). We don't know how to generalize our method to $r \geq 3$, or to other sums of the form (2), like

$$
\sum_{n=1}^{N} X_{n} X_{n+1}^{-1}
$$

or

$$
\sum_{n=1}^{N} X_{n} X_{n+1} X_{n+2}=\sum_{n=1}^{N} X_{n(n+1)(n+2)} .
$$

A natural generalisation of the sums of the form 2, using the multiplicativity of $\left(X_{m}\right)_{m \geq 1}$, gives the following problem.

224*. Open Problem. Let $\left(X_{m}\right)_{m \geq 1}$ be a random completely multiplicative function, such that $\left(X_{p}\right)_{p}$ prime are i.i.d., uniform on the unit circle. For which integer-valued polynomials $P$ and $Q$ do we have the central limit theorem:

$$
\frac{1}{\sqrt{N}} \sum_{n=1}^{N} X_{P(n)} X_{Q(n)}^{-1} \underset{N \rightarrow \infty}{\longrightarrow} \frac{\mathcal{N}_{1}+i N_{2}}{\sqrt{2}},
$$

where $\mathcal{N}_{1}, \mathcal{N}_{2}$ are independent standard Gaussian variables? In particular, does this central limt theorem occur for $P(n)=n(n+1)$ and $Q(n)=1$ ? 


\section{References}

[1] S. Chatterjee, K. Soundararajan, Random multiplicative functions in short intervals. Int. Math. Res. Notices 2012 (3) (2012), 479-492.

[2] S. Chowla, The Riemann Hypothesis and Hilbert's Tenth Problem. Gordon and Breach, New York, 1965.

[3] A. Harper, Moments of random multiplicative functions, I: low moments, better than squareroot cancellation, and critical multiplicative chaos. Preprint (2017), arXiv: 1703.06654

[4] A. Harper, A. Nikeghbali, M. Radziwill, A note on Helson's conjecture on moments of random multiplicative functions. Preprint. To appear in Analytic Number Theory in honor of Helmut Maier's 60th birthday.

[5] W. Heap, S. Lindqvist, Moments of random multiplicative functions and truncated characteristic polynomials. Preprint (2015), arXiv: 1505.03378

[6] H. Helson, Hankel Forms. Studia Math. 198 (2010), 79-84.

[7] A. Hildebrand, On consecutive values of the Liouville function. Enseign. Math. (2) 32 (1986), 219-226.

[8] K. Matomäki, M. Radziwill, T. Tao, Sign patterns of the Liouville and Möbius functions. Forum of Math., Sigma 4 (e14) (2016).

[9] J. Najnudel, On consecutive values of random completely multiplicative functions. Preprint (2017), arXiv: 1702.01470

[10] T. Tao, J. Teräväinen, Value patterns of multiplicative functions and related sequences. Forum of Math., Sigma 7 (e33) (2019).

\section{II (B) An Open Problem, by Joseph H. Silverman}

(Mathematics Department, Brown University, Providence, RI, USA)

225* A problem in (ostensibly elementary) number theory Our problem is not new, dating back to a 2004 paper of Ailon and Rudnick [2], but at the same time it is surprisingly new in the sense that one can imagine it appearing in millenia-old mathematical works from Greece, India, or China, since it involves nothing more than powers and greatest common divisors.

The initial version of the problem is simply stated: Are there infinitely many integers $n \geq 1$ such that

$$
\operatorname{gcd}\left(2^{n}-1,3^{n}-1\right)=1 ?
$$

It is natural to replace 2 and 3 with arbitrary integers $a$ and $b$, although a small amount of care is needed to avoid degenerate situations such as $a=b$, and there is the issue that $\operatorname{gcd}\left(a^{n}-1, b^{n}-1\right)$ is always divsiible by $\operatorname{gcd}(a-1, b-1)$. With these caveats, a first generalisation asks: Let $a, b \in \mathbb{Z}$ be non-zero multiplicatively independent integers, i.e., integers such that $a^{m} b^{n} \neq 1$ for all $(m, n) \neq(0,0)$. Are there infinitely many integers $n \geq 1$ such that

$$
\operatorname{gcd}\left(a^{n}-1, b^{n}-1\right)=\operatorname{gcd}(a-1, b-1) ?
$$

More generally, one may allow $a$ and $b$ to be rational numbers by defining the gcd of two rational numbers to be the ged of their numerators; and one may take $a$ and $b$ from a number field $K$ by defining

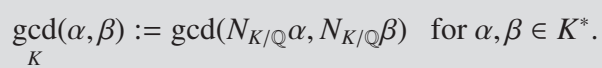

The best evidence says that there is an affirmative answer in a strong sense for characteristic 0 function fields. To avoid excessive notation, we state the result for polynomials: Let $a(T), b(T) \in$ $\mathbb{C}[T]$ be polynomials that are multiplicatively independent modulo $\mathbb{C}^{*}$. Then not only are there infinitely many $n \geq 1$ such that

$$
\operatorname{gcd}\left(a(T)^{n}-1, b(T)^{n}-1\right)=\operatorname{gcd}(a(T)-1, b(T)-1),
$$

but there is also a non-zero polynomial $c(T)$ such that

$$
\operatorname{gcd}\left(a(T)^{n}-1, b(T)^{n}-1\right) \text { divides } c(T) \text { for all } n \geq 1 .
$$

The proof by Ailon and Rudnick [2] uses a theorem due variously to Lang, Ihara, Serre and Tate [4], which says that an irreducible algebraic curve in $\mathbb{P}^{2}$ has only finitely many points with root-ofunity coordinates unless the curve is itself a translate of a torus.

Assuming that the Ailon-Rudnick question has an affirmative answer, it is natural to ask how often the ged is minimal. The fact that

$$
m\left|n \quad \Longrightarrow \quad \operatorname{gcd}\left(a^{m}-1, b^{m}-1\right)\right| \operatorname{gcd}\left(a^{n}-1, b^{n}-1\right)
$$

means that the minimal-gcd property for a composite $n$ is contingent on it being true for exponents that are factors of $n$. This suggests restricting it to prime exponents, which leads to the next question: What is the densisty (if it exists) of the set

$$
\left\{p \text { prime }: \operatorname{gcd}\left(a^{p}-1, b^{p}-1\right)=\operatorname{gcd}(a-1, b-1)\right\} ?
$$

Experiments suggest that the density is large, but they are inconclusive as to whether one should expect density 1 , or density $1-\delta$ for some small $\delta=\delta(a, b)>0$.

The strong result over function field raises the question of how large $\operatorname{gcd}\left(2^{n}-1,3^{n}-1\right)$ can be as $n \rightarrow \infty$. The function field bound is uniform in $n$, but there is no such bound for $\mathbb{Q}$, since taking $n=p-1$ with $p \geq 5$ prime, Fermat's little theorem tells us that $\operatorname{gcd}\left(2^{p-1}-1,3^{p-1}-1\right)$ is divisible by $p$. Thus, any lower bound for $\operatorname{gcd}\left(2^{n}-1,3^{n}-1\right)$ must grow at least linearly as a function of $n$, but this is far from the truth, since in fact any lower bound must grow almost exponentially: There is a constant $C=C(a, b)>0$ such that

$\log \operatorname{gcd}\left(a^{n}-1, b^{n}-1\right) \geq n^{C / \log \log n} \quad$ for infinitely many $n \geq 1$.

Bugeaud, Corvaja, and Zannier [3] noted that this follows from an analytic estimate of Adelman-Pomerance-Rumely [1, Proposition 10] that was used to prove the validity of an almost-lineartime primality test.

Thus there exists a subsequence of $\operatorname{gcd}\left(a^{n}-1, b^{n}-1\right)$ that grows almost exponentially in $n$. This raises the question of whether the growth can be fully exponential. The answer is no, as proven by Bugeaud, Corvaja, and Zannier [3]: Let $a, b \in \mathbb{Z}$ be non-zero and multiplicatively independent. Then

$$
\lim _{n \rightarrow \infty} \frac{\log \operatorname{gcd}\left(a^{n}-1, b^{n}-1\right)}{n}=0 .
$$

The proof is an intricate application of Schmidt's subspace theorem, which in turn is a higher-dimensional version of Roth's theorem on Diophantine approximation. As such, it is ineffective, in the sense that one cannot write down an explicit $n_{0}(\varepsilon)$ such that the fraction in the limit is smaller than $\varepsilon$ for all $n \geq n_{0}(\varepsilon)$. (We mention in passing for function fields over finite fields, e.g., for $a(T), b(T) \in \mathbb{F}_{p}[T]$, the Bugeaud-Corvaja-Zannier limit is false, even if one restricts $n$ to lie in a fixed congruence class modulo $p$; see [5].)

In fancier terms, the $n$-power map is an endomorphism of the multiplicative group, the pair $(a, b)$ is a point in $\mathbb{G}_{m}^{2}(\mathbb{Q})$ whose powers are Zariski dense, and $\operatorname{gcd}\left(a^{n}-1, b^{n}-1\right)$ is a measure of the arithmetic distance from $(a, b)^{n}$ to the identity $(1,1)$. This viewpoint allows us to reformulate the Ailon-Rudnick question for other (commutative) algebraic groups. Roughly speaking, for 
any algebraic variety $V / \mathbb{Q}$, we fix a model over $\mathbb{Z}$ and define the arithmetic distance between points $P, Q \in V(\mathbb{Q})$ to be

$$
\operatorname{gcd}_{V}(P, Q):=\prod_{p \text { prime }}(p \text {-adic distance from } P \text { to } Q)^{-1} .
$$

Then we ask: Let $G / \mathbb{Q}$ be a semi-abelian variety of dimension at least 2 , i.e., $G$ is the extension of an abelian variety by a torus, and let $P \in G(\mathbb{Q})$ be a point generating a subgroup $\mathbb{Z} P$ that is Zariski dense in $G$. Are there infinitely many $n \geq 1$ such that

$$
\operatorname{gcd}_{V}(n P, O)=\operatorname{gcd}_{V}(P, O) ?
$$

Specialising to $G=E_{1} \times E_{2}$ a product of elliptic curves, we get questions about $\operatorname{gcd}\left(A_{n}, B_{n}\right)$, where $A_{n}$ and $B_{n}$ are independent elliptic divisibiilty sequences. In this setting, neither the AilonRudnick question nor the analogue of the Bugeaud-CorvajaZannier limit is known, although the latter is a consequence of Vojta's conjecture applied to the blow-up of $E_{1} \times E_{2}$ at the identity.

\section{References}

[1] Leonard M. Adleman, Carl Pomerance, and Robert S. Rumely, On distinguishing prime numbers from composite numbers. Ann. of Math. (2) 117 (1983), 173-206.

[2] Nir Ailon and Zéev Rudnick, Torsion points on curves and common divisors of $a^{k}-1$ and $b^{k}-1$. Acta Arith. 113 (2004), 31-38.

[3] Yann Bugeaud, Pietro Corvaja, and Umberto Zannier, An upper bound for the G.C.D. of $a^{n}-1$ and $b^{n}-1$. Math. Z. 243 (2003), 79-84.

[4] Serge Lang, Division points on curves. Ann. Mat. Pura Appl. (4) 70 (1965), 229-234.

[5] Joseph H. Silverman, Common divisors of $a^{n}-1$ and $b^{n}-1$ over function fields. New York J. Math. 10 (2004), 37-43.

\section{III (A) Solutions}

211. Recall that a smooth function $u: \mathbf{R}^{2} \rightarrow \mathbf{R}$ is called harmonic if

$$
\Delta u(x, y):=\frac{\partial^{2} u}{\partial x^{2}}(x, y)+\frac{\partial^{2} u}{\partial y^{2}}(x, y)=0, \text { for any }(x, y) \in \mathbf{R}^{2} .
$$

Determine all harmonic polynomials in two real variables.

(Giovanni Bellettini, Dipartimento di Ingegneria dell'Informazione e Scienze Matematiche, Siena, Italia, and ICTP International Centre for Theoretical Physics, Mathematics Section, Trieste, Italy)

Solution by the proposer. Let $P(x, y)$ be a harmonic polynomial of degree $n \geq 0$ in the real variables $x$ and $y$; since the cases $n=0$ and $n=1$ are trivial, we shall assume $n \geq 2$. The first step is to show that we can reduce to the case when the polynomial is homogeneous. Indeed, we can always write $P$ as the sum of its homogeneous components:

$$
P=P_{0}+\cdots+P_{n}
$$

where, for any $i \in\{0, \ldots, n\}$, the polynomial $P_{i}$ is homogeneous of degree $i$, that is

$$
P_{i}(\lambda x, \lambda y)=\lambda^{i} P_{i}(x, y)
$$

for any $\lambda \in \mathbf{R}$ and any $(x, y) \in \mathbf{R}^{2}$. Since $\Delta$ acts linearly, we have

$$
0=\Delta P=\Delta P_{0}+\cdots+\Delta P_{n} .
$$

We observe now that $\Delta P_{i}=0$ for any $i=0, \ldots, n$. Indeed, let $j, k \in\{0, \ldots, n\}, 2 \leq j<k$, and suppose that $0=\Delta P_{j}+\Delta P_{k}$. Set $\psi_{j}:=\Delta P_{j}, \psi_{k}:=\Delta P_{k}$. By a direct computation, $\psi_{j}$ is a $(j-2)$ homogenous polynomial and $\psi_{k}$ is a $(k-2)$-homogenous polynomial. For any $\lambda \in \mathbf{R}$ and any $(x, y) \in \mathbf{R}^{2}$ we then have

$$
\begin{aligned}
0 & =\psi_{j}(\lambda x, \lambda y)+\psi_{k}(\lambda x, \lambda y)=\lambda^{j-2} \psi_{j}(x, y)+\lambda^{k-2} \psi_{k}(x, y) \\
& =\lambda^{j-2}\left(\lambda^{k-j}+1\right) \psi_{j}(x, y) .
\end{aligned}
$$

Since this equality must be valid for any $\lambda \in \mathbf{R}$, we deduce that $\psi_{j}$ must be identically zero. This observation shows therefore that

$$
\Delta P_{i}=0 \quad \forall i \in\{2, \ldots, n\},
$$

and hence we can always reduce to the case when our polynomial is $n$-homogeneous, $n \geq 2$,

$$
P_{n}(x, y)=a_{n, 0} x^{n}+a_{n-1,1} x^{n-1} y+\cdots+a_{1, n-1} x y^{n-1}+a_{0, n} y^{n} .
$$

We have to determine the $n+1$ coefficients $a_{n, 0}, a_{n-1,1}, \ldots, a_{1, n-1}, a_{0, n}$ in such a way that $\Delta P_{n}=0$. A direct computation shows that the coefficient of the generic monomial $x^{j} y^{k}$ of $\Delta P_{n}$, with $j+k=n-2$, is given by

$$
(j+2)(j+1) a_{j+2, k}+(k+2)(k+1) a_{j, k+2} .
$$

Using the expressions in (3), we obtain a homogeneous linear system of $(n-1)$ equations in $(n+1)$ unknowns; it is not difficult to check that the system has (maximal) rank $n-1$. Therefore, the subspace of solutions has dimension two. A possible choice of a basis generating the $n$-homogeneous harmonic polynomials is given by

$$
\operatorname{Re}\left(z^{n}\right)=\sum_{\substack{k=0, \ldots, n \\
k \text { even }}}\left(\begin{array}{l}
n \\
k
\end{array}\right)(-1)^{\frac{k}{2}} x^{n-k} y^{k}, \quad \operatorname{Im}\left(z^{n}\right)=\sum_{\substack{k=0, \ldots, n \\
k \text { odd }}}\left(\begin{array}{l}
n \\
k
\end{array}\right)(-1)^{\frac{k-1}{2}} x^{n-k} y^{k}
$$

where $z=x+i y \in \mathbb{C}$. Indeed, it is sufficient to check that $\operatorname{Re}\left(z^{n}\right)$ and $\operatorname{Im}\left(z^{n}\right)$ do not have proportional coefficients, and to recall that $z \in \mathbb{C} \rightarrow z^{n} \in \mathbb{C}$ is entire holomorphic, so that $\operatorname{Re}\left(z^{n}\right)$ and $\operatorname{Im}\left(z^{n}\right)$ are harmonic in $\mathbb{R}^{2}$.

Also solved by Sotirios E. Louridas (Athens, Greece), George Miliakos (Sparta, Greece) and Socratis Varelogiannis (France)

212 Reaction-diffusion systems of the form

$$
u_{t}=D u_{x x}+g(u)+\mu M u, \quad(x, t) \in \mathbb{R} \times(0, \infty),
$$

where

$$
\begin{array}{r}
u(x, t) \in \mathbb{R}^{n}, g_{i}(u)=r_{i} u_{i}\left(1-\sum_{j=1}^{n} \alpha_{j} u_{j}\right), r_{i}, \alpha_{i}>0, \\
i=1, \ldots, n, \mu>0,
\end{array}
$$

and $D$ and $M$ are constant $n \times n$ matrices such that $D$ is positivedefinite diagonal and $M$ has strictly positive off-diagonal elements and zero column sums, arise in the modelling of the population densities of $n$ phenotypes of a species that diffuse, compete both within a phenotype and with other phenotypes, and may mutate from one phenotype to another. Denoting the Perron-Frobenius eigenvalue of a matrix $Q$ by $\eta_{P F}[Q]$ and assuming that the $n$ phenotypes spread together into an unoccupied spatial region at the 
$\mu$-dependent speed

$$
c(\mu):=\inf _{\beta>0} \eta_{P F}\left[\beta D+\beta^{-1}\left(\operatorname{diag}\left(r_{1}, \ldots, r_{n}\right)+\mu M\right)\right],
$$

which is determined by the linearisation of the reaction-diffusion system about the extinction steady state $u=(0, \ldots, 0) \in \mathbb{R}^{n}$, prove that spreading speed $c(\mu)$ is a non-increasing function of $\mu$.

(Elaine Crooks, Department of Mathematics,

College of Science, Swansea University,

Swansea, UK)

Solution by the proposer. Let $\mu>\mu_{0}>0$, denote the zero $n \times n$ matrix by $Z$, and define

$$
P:=\bar{\beta}^{2} D+\operatorname{diag}\left(r_{1}, \ldots, r_{n}\right)-c\left(\mu_{0}\right) I,
$$

where $\bar{\beta}$ is such that the infimum in the definition of $c\left(\mu_{0}\right)$ is attained at $\beta=\bar{\beta}$. Then

$$
\begin{aligned}
\eta_{P F}\left[P+\mu_{0} M\right] & =\bar{\beta} \eta_{P F}\left[\bar{\beta} D+\bar{\beta}^{-1}\left(\operatorname{diag}\left(r_{1}, \ldots, r_{n}\right)+\mu_{0} M\right)-c\left(\mu_{0}\right) I\right] \\
& =0,
\end{aligned}
$$

and by the convexity of the Perron-Frobenius eigenvalue of a matrix on its diagonal,

$$
\begin{aligned}
\eta_{P F}\left[\frac{1}{\mu} P+M\right] & \leq \frac{\mu_{0}}{\mu} \eta_{P F}\left[\frac{1}{\mu_{0}} P+M\right]+\left(1-\frac{\mu_{0}}{\mu}\right) \eta_{P F}[Z+M] \\
& =\frac{\mu_{0}}{\mu} \eta_{P F}\left[\frac{1}{\mu_{0}} P+M\right]+\left(1-\frac{\mu_{0}}{\mu}\right) \eta_{P F}[M] \\
& =0,
\end{aligned}
$$

since

$$
\eta_{P F}[M]=0 \quad \text { and } \quad \eta_{P F}\left[\frac{1}{\mu_{0}} P+M\right]=\frac{1}{\mu_{0}} \eta_{P F}\left[P+\mu_{0} M\right]=0 .
$$

Hence

$$
\eta_{P F}[P+\mu M] \leq 0
$$

which says that,

$$
\eta_{P F}\left[\bar{\beta} D+\bar{\beta}^{-1}\left(\operatorname{diag}\left(r_{1}, \ldots, r_{n}\right)+\mu M\right)\right] \leq c\left(\mu_{0}\right),
$$

and so

$$
c(\mu):=\min _{\beta>0} \eta_{P F}\left[\beta D+\beta^{-1}\left(\operatorname{diag}\left(r_{1}, \ldots, r_{n}\right)+\mu M\right)\right] \leq c\left(\mu_{0}\right) .
$$

Also solved by Mihály Bencze (Brasov, Romania), Jim Kelesis (Athens, Greece), Sotirios E. Louridas (Athens, Greece), George Miliakos (Sparta, Greece)

213. Consider the second-order PDE with non-constant coefficients,

$$
u_{x x}-x^{2} u_{y y}=0
$$

Find at least one family of solutions.

(Jonathan Fraser, School of Mathematics and Statistics, The University of St Andrews, Scotland)

Solution by the proposer. In the general form of a second-order PDE,

$$
a(x, y) u_{x x}+2 b(x, y) u_{x y}+c(x, y) u_{y y}=0,
$$

we have $a=1, b=0$ and $c=-x^{2}$. Hence, the discriminant $b^{2}-a c=x^{2}$ implying that the equation is hyperbolic for $x \neq 0$. The characteristic curves are given by

$$
\frac{d y}{d x}=\frac{b}{a} \pm \frac{1}{a} \sqrt{b^{2}-a c}= \pm x, \quad \Longrightarrow \quad y= \pm \frac{1}{2} x^{2}+\text { constant. }
$$

So the characteristic coordinates are

$$
\xi=y+\frac{1}{2} x^{2}, \quad \eta=y-\frac{1}{2} x^{2}
$$

noting

$$
\xi_{x}=x, \quad \xi_{y}=1, \quad \eta_{x}=-x, \quad \eta_{y}=1 .
$$

Hence,

$$
\begin{aligned}
u_{x} & =x u_{\xi}-x u_{\eta}, \\
u_{x x} & =\left[x\left(u_{\xi}-u_{\eta}\right)\right]_{x} \\
& =\left(u_{\xi}-u_{\eta}\right)+x\left(u_{\xi}-u_{\eta}\right)_{x} \\
& =\left(u_{\xi}-u_{\eta}\right)+x^{2}\left(u_{\xi \xi}-2 u_{\xi \eta}+u_{\eta \eta}\right) \\
u_{y} & =u_{\xi}+u_{\eta}, \\
u_{y y} & =u_{\xi \xi}+2 u_{\xi \eta}+u_{\eta \eta} .
\end{aligned}
$$

Substituting into $u_{x x}-x^{2} u_{y y}=0$, we obtain

$$
-4 x^{2} u_{\xi \eta}+\left(u_{\xi}-u_{\eta}\right)=0 \quad \Longrightarrow \quad u_{\xi \eta}=\frac{\left(u_{\xi}-u_{\eta}\right)}{4 x^{2}} .
$$

Finally, we must replace the $x$ in the equation by $\xi$ and $\eta$. From their definitions, we have

$$
\xi-\eta=x^{2},
$$

and so the canonical form of the equation is

$$
u_{\xi \eta}=\frac{\left(u_{\xi}-u_{\eta}\right)}{4(\xi-\eta)} .
$$

It turns out that this equation can be solved by assuming a solution of the form

$$
u(\xi, \eta)=f(\xi \eta)=f(t)
$$

with $t=\xi \eta$. Differentiating gives

$$
u_{\xi}=\eta f^{\prime}, \quad u_{\eta}=\xi f^{\prime}, \quad u_{\xi \eta}=f^{\prime}+\xi \eta f^{\prime \prime} .
$$

When these are substituted into the canonical form of the equation, it reduces (greatly) to a single first-order ODE for $g=f^{\prime}$,

$$
4 t g^{\prime}+5 g=0
$$

which can be solved and integrated to give

$$
f=A(\xi \eta)^{-1 / 4}+B
$$

Therefore the solution in terms of the original $x, y$ variables is

$$
u=A\left[\left(y+\frac{1}{2} x^{2}\right)\left(y-\frac{1}{2} x^{2}\right)\right]^{-1 / 4}+B=A\left(y^{2}-\frac{1}{4} x^{4}\right)^{-1 / 4}+B .
$$

This can be verified by direct differentiation. A solution exists only in the regions above $y=\frac{1}{2} x^{2}$ or below $y=-\frac{1}{2} x^{2}$, i.e., above and below the characteristics $\xi=0$ and $\eta=0$ emanating from $(0,0)$, as shown in Figure 1. 


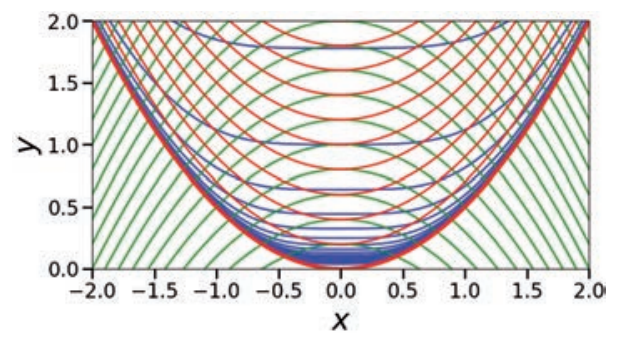

Figure 1. Illustration of the solution to the (almost) hyperbolic PDE $u_{x x}-x^{2} u_{y y}=0$. The blue curves show lines of constant $u$ (equally spaced in value). Note $u \rightarrow \infty$ approaching the critical characteristic $y=\frac{1}{2} x^{2}$ (the thick red curve). The characteristics $y=\xi-\frac{1}{2} x^{2}$ are shown in green, and the characteristics $y=\eta+\frac{1}{2} x^{2}$ are shown in red (for $\eta \geq 0$ ). Note that the characteristics and solution curves are tangent along $x=0$ where the PDE is parabolic.

Note, there are in fact many other solutions. One for example is

$$
u=a(\xi+\eta)=2 a y .
$$

Another is

$$
u=a\left(\xi^{2}+\eta^{2}\right)+b \xi \eta
$$

so long as $b=2 a / 5$. In the coordinates $x$ and $y$, this solution is

$$
u(x, y)=\frac{2 a}{5}\left(6 y^{2}+x^{4}\right) .
$$

Infinitely many other analogous solutions can be constructed assuming

$$
u(\xi, \eta)=\sum_{j=0}^{n} a_{j} \xi^{n-j} \eta^{j}
$$

for all $n>0$, and with $a_{n-j}=a_{j}$ for all $j$. It is just a matter of plugging this form into the PDE and determining relations among the coefficients $a_{j}$.

Also solved by Mihály Bencze (Brasov, Romania), Sotirios E. Louridas (Athens, Greece), George Miliakos (Sparta, Greece) and Socratis Varelogiannis (France)

\section{Let $u$ solve}

$$
\left(\Delta+200^{2} x y^{2}\right) u=1
$$

on the triangle $T=\{(x, y): 0<x<1,0<y<1-x\}$ with zero Dirichlet conditions:

$$
u(x, 0)=u(0, y)=u(x, 1-x)=0 .
$$

What are the first 10 significant digits of $u(0.1,0.2)$ ?

(Sheehan Olver, Department of Mathematics, Imperial College, London, UK)

Solution by the proposer.

\section{$-0.00321203523532$}

should be accurate to 12 digits of relative accuracy.

This problem is motivated by a similar question recently posed by A. Gopal and L. N. Trefethen on NADigest, 3 Dec 2018:

Let the domain be the $L$-shaped region in the $(x, y)$-plane consisting of $[0,2] \times[0,2]$ minus its upper-right quarter. If $u$ is harmonic in this region with $u=x^{2}$ on the boundaries, what is $u(.99, .99)$ to 8 digits?
The difficulty is that the solution has weak singularities at the corners, which limits accuracy of most numerical methods. They proposed a solution based on using fundamental solutions with cleverly chosen spacing near the corners [6], but this technique is not immediately adaptable to Problem 1, where the fundamental solutions are not known in closed form. Furthermore, the large factor introduces oscillations into the solution and ill-conditioning into standard discretisations that make the problem challenging.

To solve Problem 1 we use a recently introduced method [3], where we represent the solution in orthogonal polynomials on the triangle and construct a sparse representation of the partial differential operator. This is close to prior work on $p$-finite element methods $[1,5]$, but with the added benefit of sparsity for variable coefficients. This sparsity allows us to use very high order approximations and thereby resolve the solution to high accuracy, despite the corner singularities and oscillations.

In detail, define

$$
P_{n, k}^{(a, b, c)}(x, y):=P_{n-k}^{(2 k+b+c+1, a)}(2 x-1)(1-x)^{k} P_{k}^{(b, c)}(2 y /(1-x)-1)
$$

which are orthogonal with respect to the weight $x^{a} y^{b}(1-x-y)^{c}$ on $T$ (cf. for example [2]) and write

$$
u(x, y) \approx u_{N}(x, y)=x y z \sum_{n=0}^{N} \sum_{k=0}^{n} u_{n, k}^{N} P_{n, k}^{(1,1,1)}(x, y)
$$

where $z:=1-x-y$ and the coefficients $u_{n, k}^{N}$ are to be determined. The action of the Laplacian on this basis can be deduced in closed form by employing recurrence relationships for the orthogonal polynomials [4]. That is, the following recurrences (which follow from manipulation of 1D Jacobi polynomial relationships) can be combined to express $\frac{\mathrm{d}^{2}}{\mathrm{~d} x^{2}}\left(x y z P_{n, k}^{(1,1,1)}\right)$ in terms of $P_{n, k}^{(1,1,1)}$ :

$$
\begin{aligned}
&-(2 k+3) \frac{\mathrm{d}}{\mathrm{d} x}\left(x y z P_{n, k}^{(1,1,1)}\right)=y((k+1)(n-k+1) P_{n+1, k}^{(0,1,0)} \\
&\left.+(k+1)(n-k+1) P_{n+1, k+1}^{(0,1,0)}\right), \\
&(2 k+2)(2 n+3) y P_{n, k}^{(0,1,0)}=(+1)(n+k+2) P_{n, k}^{(0,0,0)} \\
&-(k+1)(n-k) P_{n, k+1}^{(0,0,0)} \\
&-(k+1)(n-k+1) P_{n+1, k}^{(0,0,0)} \\
&+(k+1)(n+k+3) P_{n+1, k+1}^{(0,0,0)}, \\
&(2 k+1) \frac{\mathrm{d}}{\mathrm{d} x} P_{n, k}^{(0,0,0)}=(n+2)(k+1) P_{n-1, k}^{(1,0,1)}, \\
&(2 n+4)(2 k+2) P_{n, k}^{(1,0,1)}=(n+k+4)(k+2) P_{n, k}^{(1,1,1)} \\
&-(n-k+1)(k+2) P_{n-1, k}^{(1,1,1)} \\
&+(k+1)(n+k+2) P_{n-1, k-1}^{(1,1,1)} \\
&-(k+1)(n-k+1) P_{n, k-1}^{(1,1,1)} .
\end{aligned}
$$

The following can be combined to express $\frac{\mathrm{d}^{2}}{\mathrm{~d} y^{2}}\left(x y z P_{n, k}^{(1,1,1)}\right)$ also in terms of $P_{n, k}^{(1,1,1)}$ :

$$
\begin{aligned}
\frac{\mathrm{d}}{\mathrm{d} y}\left(x y z P_{n, k}^{(1,1,1)}\right) & =-(k+1) x P_{n+1, k+1}^{(1,0,0)}, \\
(2 n+3) x P_{n, k}^{(1,0,0)} & =(n-k+1)\left[P_{n, k}^{(0,0,0)}+P_{n+1, k}^{(0,0,0)}\right], \\
\frac{\mathrm{d}}{\mathrm{d} y} P_{n, k}^{(0,0,0)} & =(k+1) P_{n-1, k-1}^{(0,1,1)}, \\
(2 n+4) P_{n, k}^{(0,1,1)} & =(n+k+4) P_{n, k}^{(1,1,1)}+(n+k+3) P_{n-1, k}^{(1,1,1)} .
\end{aligned}
$$

Thus $\Delta\left(x y z P_{n, k}^{(1,1,1)}\right)$ has a sparse expansion in $P_{n, k}^{(1,1,1)}$, which can be found in closed form. 

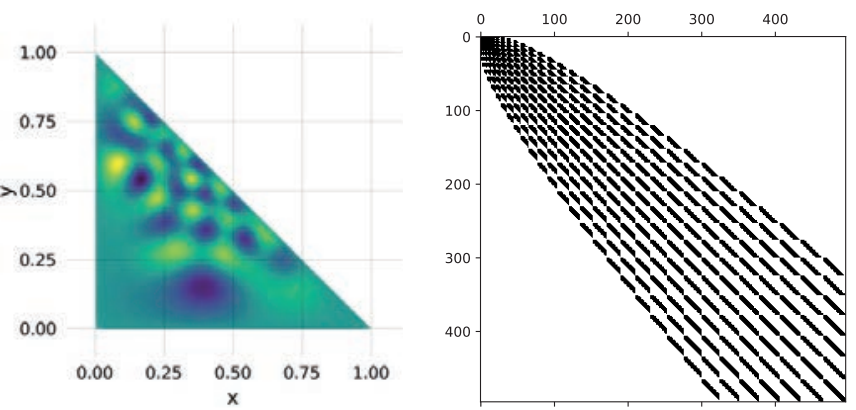

Figure 2. The solution (left). The sparsity of the discretisation $L^{N}$ for $N=30$ (right).

We similarly can construct a sparse expression for multiplication by $x y^{2}$, using the following:

$$
\begin{aligned}
& (2 n+5) x P_{n, k}^{(1,1,1)}=(n-k+1) P_{n, k}^{(0,1,1)}+(n-k+1) P_{n+1, k}^{(0,1,1)}, \\
& (2 n+4) P_{n, k}^{(0,1,1)}=(n+k+4) P_{n, k}^{(1,1,1)}+(n+k+3) P_{n-1, k}^{(1,1,1)}, \\
& (2 k+3)(2 n+5) y P_{n, k}^{(1,1,1)}=(k+1)(n+k+3) P_{n, k}^{(1,0,1)} \\
& -(k+1)(n-k+1) P_{n, k+1}^{(1,0,1)} \\
& -(k+1)(n-k+1) P_{n+1, k}^{(1,0,1)} \\
& +(k+1)(n+k+5) P_{n+1, k+1}^{(1,0,1)}, \\
& (2 n+4)(2 k+2) P_{n}^{(1,0,1)}=(n+k+4)(k+2) P_{n, k}^{(1,1,1)} \\
& -(n-k+1)(k+2) P_{n-1, k}^{(1,1,1)} \\
& +(k+1)(n+k+2) P_{n-1, k-1}^{(1,1,1)} \\
& -(k+1)(n-k+1) P_{n, k-1}^{(1,1,1)}, \\
& (2 k+3)(2 n+5) z P_{n, k}^{(1,1,1)}=(k+1)(n+k+3) P_{n, k}^{(1,1,0)} \\
& +(k+1)(n-k+1) P_{n, k+1}^{(1,1,0)} \\
& -(k+1)(n-k+1) P_{n+1, k}^{(1,1,0)} \\
& -(k+1)(n+k+5) P_{n+1, k+1}^{(1,1,0)}, \\
& (2 n+4)(2 k+2) P_{n}^{(1,1,0)}=(n+k+4)(k+2) P_{n, k}^{(1,1,1)} \\
& -(n-k+1)(k+2) P_{n-1, k}^{(1,1,1)} \\
& -(k+1)(n+k+2) P_{n-1, k-1}^{(1,1,1)} \\
& +(k+1)(n-k+1) P_{n, k-1}^{(1,1,1)} \text {. }
\end{aligned}
$$

Recurrence relationships induce an operator on matrix coefficients, that is, there exists a block $(N+6) \times N$ matrix $L^{N}$ so that

$$
\begin{aligned}
\Delta u_{N}(x, y) & =\Delta\left[x y z\left(P_{0,0}^{(1,1,1)}, P_{1,0}^{(1,1,1)}, \ldots, P_{N, N}^{(1,1,1)}\right)\left(\begin{array}{c}
u_{0,0}^{N} \\
\vdots \\
u_{N, N}^{N}
\end{array}\right)\right] \\
& =\left(P_{0,0}^{(1,1,1)}, P_{1,0}^{(1,1,1)}, \ldots, P_{N+1, N+1}^{(1,1,1)}\right) L^{N}\left(\begin{array}{c}
u_{0,0}^{N} \\
\vdots \\
u_{N, N}^{N}
\end{array}\right)
\end{aligned}
$$

Using this we arrive at a linear system:

$$
L^{N} \boldsymbol{u}^{N}=\boldsymbol{e}_{0}
$$

where $\boldsymbol{u}^{N}$ is best interpreted as a block-vector with blocks $\boldsymbol{u}_{n}^{N}=$ $\left(u_{n, 0}^{N}, \ldots, u_{n, n}^{N}\right)^{\top}$, and $L^{N}$ is a block-matrix whose entries are determined by $\Delta\left(x y z P_{n, k}^{(1,1,1)}\right)$ using the recurrences. This is a sparse linear system, see the right-hand side of Figure 1, and can be solved efficiently, either in $O\left(N^{4}\right)$ operations using a block-QR decomposition or in $O\left(N^{3}\right)$ operations using sparse direct methods, as implemented in UMFPack. The result was determined using $N=1000$, which matched the calculation with $N=999$ to an absolute accuracy of $5.5 \times 10^{-17}$.

\section{References}

[1] Beuchler, S., Schoeberl, J., New shape functions for triangular p-FEM using integrated Jacobi polynomials. Numer. Math. 103 (2006), 339366.

[2] Dunkl, C. F., Xu, Y., Orthogonal Polynomials of Several Variables. Cambridge University Press, 2014.

[3] Olver, S., Townsend, A., Vasil, G., A sparse spectral method on triangles. Preprint arXiv: 1902.04863, 2019.

[4] Olver, S., Townsend, A., Vasil, G., Recurrence relations for orthogonal polynomials on a triangle. ICOSAHOM 2018, to appear.

[5] Li, H., Shen, J., Optimal error estimates in Jacobi-weighted Sobolev spaces for polynomial approximations on the triangle. Maths Comp. 79 (2010), 1621-1646.

[6] Gopal, A., Trefethen, L. N., New Laplace and Helmholtz solvers. Proc. Nat. Acad. Sci., 116 (2019), 10223-10225.

Also solved by Mihály Bencze (Brasov, Romania) and Socratis Varelogiannis (France)

215. Let $u$ be an entire harmonic function in $\mathbf{R}^{n}$, satisfying $u(x) \geq-c\left(1+|x|^{m}\right)$ for some constants $c>0$ and $m \in \mathbb{N}$. Show that $u$ is a polynomial of degree less or equal to $m$.

(Gantumur Tsogtgerel, McGill University, Department of Mathematics and Statistics, Montreal, Canada)

Solution by the proposer. Let us first derive an upper bound on $u$. To this end, let $r>0$ and let

$$
v(x)=u(x)+c\left(1+r^{m}\right) .
$$

Then $v$ is harmonic, and $v \geq 0$ in $B(0, r)$, where $B(0, r) \subset \mathbf{R}^{n}$ is the closed ball of radius $r>0$, centred at the origin. Now, pick $x \in \mathbf{R}^{n}$ with $|x|=r / 2$, and invoke the mean value property to get

$$
v(0)=\frac{1}{|B(0, r)|} \int_{B(0, r)} v \geq \frac{1}{|B(0, r)|} \int_{B(x, r / 2)} v=\frac{|B(x, r / 2)|}{|B(0, r)|} v(x),
$$

where $B(x, r / 2)$ is the closed ball of radius $r / 2$, centred at $x$. This yields

$$
u(x) \leq v(x) \leq 2^{n} v(0)=2^{n}\left(u(0)+c+c r^{m}\right),
$$

and since $r$ was arbitrary, we conclude

$$
u(x) \leq 2^{n}\left(u(0)+c+c 2^{m}|x|^{m}\right), \quad c \in \mathbf{R}^{n} .
$$

Combining it with the lower bound $u(x) \geq-c\left(1+|x|^{m}\right)$, we infer

$$
|u(x)| \leq A+B|x|^{m},
$$

for some constants $A$ and $B$. Finally, the standard derivative estimate

$$
\left|\partial^{\alpha} u(x)\right| \leq \frac{M(n,|\alpha|)}{r^{|\alpha|}} \sup _{B(x, r)}|u|,
$$

for harmonic functions finishes the job.

Also solved by Mihály Bencze (Brasov, Romania), John N. Daras (Athens, Greece), and Jim Kelesis (Athens, Greece) 
216. Let $f:[0, \infty) \rightarrow(0, \infty)$ be a continuous function satisfying $f(x) \rightarrow 0$ as $x \rightarrow \infty$, and let

$$
\Omega=\left\{(x, y) \in \mathbf{R}^{2}: x>0,0<y<f(x)\right\} .
$$

Exhibit an unbounded function $u$ in $\Omega$, such that $u \in H^{k}(\Omega)$ for all $k \geq 0$. Here $H^{k}(\Omega)$ is the standard Sobolev space of functions whose partial derivatives of all orders up to $k$ are square integrable.

(Gantumur Tsogtgerel, McGill University, Department of Mathematics and Statistics, Montreal, Canada)

Solution by the proposer. Fix a nontrivial smooth "bump" function $\varphi: \mathbf{R} \rightarrow[0, \infty)$, supported in the interval $(0,1)$, and let

$$
u(x, y)=\sum_{n=1}^{\infty} n \varphi\left(x-a_{n}\right), \quad(x, y) \in \Omega,
$$

where $\left\{a_{n}\right\}$ is an increasing sequence of numbers, to be determined below. It is clear that $u$ is well-defined and unbounded as long as $\left\{a_{n}\right\}$ grows sufficiently fast.
Let us compute the $L^{2}$-norm of $u$. Assuming that $\left\{a_{n}\right\}$ grows sufficiently fast, we have

$$
\int_{\Omega}|u|^{2}=\sum_{n=1}^{\infty} n \int_{a_{n}}^{a_{n}+1} f(x)\left|\varphi\left(x-a_{n}\right)\right|^{2} d x=\sum_{n=1}^{\infty} n \int_{0}^{1} f\left(t+a_{n}\right)|\varphi(t)|^{2} d t,
$$

which will be finite if we choose, e.g., $a_{n}$ so large that $f(x)<n^{-3}$ for all $x>a_{n}$. It is not difficult to see that the same condition works for the derivatives as well.

Also solved by Mihály Bencze (Brasov, Romania), John N. Daras (Athens, Greece) and Socratis Varelogiannis (France)

We would like for you to submit solutions to the proposed problems and ideas on the open problems. Send your solutions by email to Michael Th. Rassias, Institute of Mathematics, University of Zürich, Switzerland, michail.rassias@math.uzh.ch.

We also solicit your new problems with their solutions for the next "Solved and Unsolved Problems" column, which will be devoted to Algebra. 